\title{
Desempenho produtivo de cabritos alimentados com glicerina bruta
}

\author{
Productive performance of goat kids fed of crude glycerin
}

\section{Diego Amorim dos Santos ${ }^{\mathrm{I}}$ Henrique Nunes Parente $^{\mathrm{I}^{*}}$ Michelle de Oliveira Maia Parente $^{\mathrm{I}}$ Karlyene Sousa da Rocha ${ }^{I}$ Evandro Maia Ferreira ${ }^{\text {II }}$ Arnaud Azevêdo Alves ${ }^{\text {III }}$}

\section{RESUMO}

Avaliou-se o desempenho produtivo de cabritos mestiços terminados em confinamento, alimentados com dietas contendo glicerina bruta (0, 4, 8 e $12 \%$ na MS). Vinte cabritos mestiços foram utilizados em blocos completos, casualizados com quatro tratamentos e cinco repetições, para determinação do ganho médio diário (GMD), consumo de matéria seca (CMS) e nutrientes (PB, FDN, EE) e comportamento ingestivo. Para o ensaio de digestibilidade, foram utilizados quatro caprinos alocados em delineamento em quadrado latino, para determinação da digestibilidade da MS e nutrientes (PB, FDN, $E E)$. A adição de glicerina bruta não interferiu $(P>0,05)$ no $G M D$ e no CMS e demais nutrientes. O tempo de ingestão aumentou com o incremento de glicerina bruta nas dietas. Não houve diferença significativa $(P>0,05)$ dos tratamentos sobre o tempo gasto na ruminação e digestibilidade da $M S, P B$ e FDN, contudo, houve efeito quadrático para a digestibilidade do EE. Recomendase a inclusão em até $12 \%$ de glicerina bruta para cabritos em terminação, em situações de oscilação do preço do milho, sem alteração nos parâmetros produtivos avaliados.

Palavras-chave: confinamento, consumo, desempenho, glicerol.

\section{ABSTRACT}

This study was conducted to evaluate the productive performance of crossbred goats finished in feedlots fed diets containing increasing levels of crude glycerin $(0,4,8$ and $12 \%$, DM basis). Twenty crossbred goat kids were used in a randomized complete block design with 4 treatments and 5 replications to determine average daily gain $(A D G)$, dry matter intake (DMI) and nutrients (CP, NDF, EE) and ingestive behavior. For digestibility trial, four castrated male goats were used in a Latin Square Design to determine the DM and nutrients digestibility. Crude glycerin did not affect $(P>0.05)$ the $A D G, D M I$ and nutrients intake. Eating time increased linearly $(P<0.05)$ with crude glycerin addition. Rumination time and dry matter and nutrients digestibility were unaffected by crude glycerin addition. However was a quadratic effect $(P<0.05)$ to EE digestibility. It is recommended adding up to $12 \%$ crude glycerin to finishing kids, in situations of high price of corn, without changes in production parameters evaluated.

Key words: feedlot, glycerol, intake, performance.

\section{INTRODUÇ̃̃O}

Com o propósito de viabilizar o confinamento na região Nordeste, é comum à utilização de coprodutos da agroindústria na composição das dietas, uma vez que o custo com a alimentação é responsável por grande parte dos gastos neste sistema. Dentre estes coprodutos, a glicerina bruta aparece como uma opção na formulação de dietas, podendo substituir o milho, quando o preço representar até $70 \%$ do preço do milho (LAGE et al., 2014).

A cada $9 \mathrm{~kg}$ do biodiesel produzido, há disponibilidade de $1 \mathrm{~kg}$ de glicerina bruta (DASARI et al., 2005), evidenciando o volume de resíduo que esta indústria produzirá nos próximos anos. $\mathrm{O}$ destino à glicerina oriunda da indústria do biodiesel é um fator a ser analisado pelo setor dos biocombustíveis. A Food and Drug Administration (FDA, 21CFR582.1320,

'Programa de Pós-graduação em Ciência Animal, Universidade Federal do Maranhão (UFMA), Campus Chapadinha, BR 222, Km 04, S/N, Bairro Boa Vista, 65500-000, Chapadinha, MA, Brasil. E-mail: hnparente@hotmail.com. *Autor para correspondência.

"Departamento de Zootecnia, Universidade Estadual de Ponta Grossa (UEPG), Ponta Grossa, PR, Brasil.

IIIPrograma de Pós-graduação em Ciência Animal, Universidade Federal do Piauí (UFPI), Teresina, PI, Brasil. Recebido 07.04.14 Aprovado 12.08.14 Devolvido pelo autor 09.09.14 CR-2014-0536.R2 
2013) atesta a segurança do uso da glicerina bruta na nutrição de ruminantes, ressalvando apenas o teor de metanol presente no produto, que não deve ultrapassar a $150 \mathrm{mg} \mathrm{kg}^{-1}$.

De acordo com ELAM et al. (2008), por ser hidroscópica, a glicerina estimula a ingestão do concentrado pelos animais e, por possuir aroma suave e sabor adocicado, melhora a palatabilidade da ração, favorecendo o seu consumo. Metabolicamente, o glicerol pode ter duas destinações: a absorção imediata pelo epitélio ruminal e posterior transporte ao fígado para a gliconeogênese ou a fermentação no ambiente ruminal, originando ácido propiônico, que seguirá a mesma rota de absorção e servirá, também, a via gliconeogênica. Assim, é grande o potencial energético da glicerina para ruminantes, funcionando como substrato neoglicogênico (KREHBIEL, 2008).

Com objetivo de avaliar este coproduto da fabricação do biodiesel, alguns trabalhos já foram realizados com ovinos (ÁVILA-ESTAGNO et al., 2012; LAGE et al., 2014), entretanto, não há estudos comprovando a eficiência deste nas dietas de caprinos. Nesse contexto, objetivou-se avaliar o desempenho produtivo de cabritos alimentados com dietas contendo glicerina bruta terminados em confinamento.

\section{MATERIAL E MÉTODOS}

O experimento foi conduzido no Setor de Pequenos Ruminantes do Centro de Ciências Agrárias e Ambientais da Universidade federal do Maranhão, município de Chapadinha, MA.

Foram utilizados 20 cabritos machos, castrados, mestiços, com três meses de idade, peso médio inicial de $17,06 \pm 2,16 \mathrm{~kg}$, para o ensaio de desempenho e comportamento ingestivo.

Os animais foram vermifugados, identificados e alojados em baias individuais de $1,5 \mathrm{~m}^{2}$, providas de comedouro e bebedouros, localizadas em galpão coberto de alvenaria com piso de concreto, onde permaneceram durante todo o período experimental, compreendendo um período inicial de 14 dias destinados à adaptação às dietas e instalações e 51 dias destinados à coleta de dados.

As dietas foram formuladas para serem isonitrogenadas, calculadas de acordo com as exigências prescritas pelo NRC (2007) para caprinos com potencial de GMD de $150 \mathrm{~g}$ (Tabela 1), caracterizando quatro tratamentos, com cinco repetições em um delineamento em blocos completos casualizados. As mesmas foram fornecidas inteiramente na parte da manhã, às $8 \mathrm{~h}$, proporcionando sobra média de $10 \%$ da matéria

Tabela 1 - Proporção dos ingredientes e composição química das dietas.

\begin{tabular}{|c|c|c|c|c|}
\hline & G0 & G4 & G8 & G12 \\
\hline \multicolumn{5}{|c|}{ - } \\
\hline Feno de Tifton-85 & 30,0 & 30,0 & 30,0 & 30,0 \\
\hline Milho em grão triturado & 53,0 & 47,0 & 42,0 & 37,8 \\
\hline Farelo de soja & 14,3 & 16,3 & 17,3 & 17,5 \\
\hline Glicerina bruta & 0,0 & 4,0 & 8,0 & 12,0 \\
\hline Calcário & 1,2 & 1,2 & 1,2 & 1,2 \\
\hline Mistura mineral $^{2}$ & 1,5 & 1,5 & 1,5 & 1,5 \\
\hline Matéria orgânica & 95,88 & 95,60 & 95,63 & 95,59 \\
\hline Matéria seca & 85,64 & 85,63 & 85,72 & 85,81 \\
\hline Proteína bruta & 13,60 & 14,09 & 14,17 & 13,93 \\
\hline Fibra em detergente neutro & 32,34 & 31,98 & 31,57 & 31,14 \\
\hline Extrato etéreo & 2,83 & 2,67 & 2,53 & 2,41 \\
\hline Cinzas & 4,12 & 4,40 & 4,37 & 4,41 \\
\hline Carboidratos totais & 79,45 & 78,84 & 78,93 & 79,25 \\
\hline Carboidratos não fibrosos & 41,44 & 41,63 & 43,21 & 43,72 \\
\hline Nutrientes Digestíveis Totais & 87,45 & 89,23 & 88,05 & 87,15 \\
\hline
\end{tabular}

${ }^{1} \mathrm{G} 0, \mathrm{G} 4, \mathrm{G} 8$, G12: adição de $0,4,8$ e $12 \%$ de glicerina bruta na dieta, respectivamente. ${ }^{2}$ Composição: Ca $13,4 \% ; \mathrm{P} 7,5 \%$; Mg 1\%; S 7\%. Na $14,5 \%$; Mn $1100 \mathrm{mg} \mathrm{kg}^{-1}$; Fe $500 \mathrm{mg} \mathrm{kg}^{-1}$. 
seca fornecida por dia. Água e sal mineral estiveram disponíveis à vontade. As sobras do dia anterior eram pesadas antes do fornecimento da dieta para cálculo do consumo médio diário.

Aproximadamente, $10 \%$ das sobras de cada semana foram amostradas e compostas por tratamento. As amostras das sobras, ingredientes e de cada partida de ração foram conservadas a $-20^{\circ} \mathrm{C}$ para serem realizadas posteriores análises bromatológicas. As dietas utilizadas foram formuladas com relação volumoso:concentrado 30:70 e as partículas tamanho de $30 \mathrm{~mm}$, sendo o volumoso constituído por feno de capim Tifton-85.

Os animais foram pesados no primeiro e último dia do período experimental, sempre no período da manhã, após jejum de sólidos de 16 horas para posterior cálculo do ganho de peso médio diário (GMD). A conversão alimentar da MS (CAMS) foi calculada em função do consumo e do desempenho animal, conforme a equação: $\mathrm{CA}=(\mathrm{IDMS} / \mathrm{GMD})$. IDMS = ingestão diária de matéria seca $\left(\mathrm{kg} \mathrm{MS} \mathrm{dia}{ }^{-1}\right)$ e GMD = ganho médio diário $\left(\mathrm{kg} \mathrm{dia}^{-1}\right)$.

Para a análise do comportamento ingestivo, as avaliações consistiram em anotações das atividades do animal, a saber: ingestão, ruminação e ócio, obtidas através do método scan sampling, realizado em intervalos iguais de 5 minutos durante 24 horas ininterruptas no $24^{\circ}$ e $46^{\circ}$ dias de confinamento, seguindo a metodologia descrita por AZEVEDO et al. (2013). O tempo total em minutos por dia gasto por cada animal em cada atividade foi calculado multiplicando-se o número total de observações por cinco. As atividades de ruminação, mastigação e ingestão foram expressas em minutos dia $^{-1}$, minutos $\mathrm{g}^{-1}$ de MS ingerida e minutos. $\mathrm{g}^{-1}$ de FDN ingerida, expressos pela razão entre o tempo diário de ingestão e ruminação e a quantidade do nutriente em questão consumida diariamente.

Para o ensaio da digestibilidade, utilizaramse quatro animais com peso inicial de $22,4 \pm 3,06 \mathrm{~kg}$, os quais foram alojados em gaiolas metabólicas para avaliação das dietas pelo método de coleta total de fezes in vivo. O ensaio foi realizado com duração de 40 dias, divididos em quatro períodos, sendo compreendidos em sete dias para adaptação e três dias para coleta dos dados.

Todos os dias do período de colheita, as rações foram pesadas em balança eletrônica com precisão de 5 gramas e ofertadas à vontade às $8 \mathrm{~h}$ da manhã. A quantidade de ração ofertada foi ajustada com base no consumo de ração obtido durante o período de adaptação dos animais, não se permitindo sobras superiores a $10 \%$ da quantidade ofertada.
Durante os quatro dias do período de colheita, às $7 \mathrm{~h} 30 \mathrm{~min}$ da manhã, foram pesadas as sobras de ração, para obtenção do consumo de matéria seca por animal, e a quantidade total de fezes produzidas em 24 horas. Para a colheita de fezes, utilizaram-se arreios com bolsa coletora de fezes, com o objetivo de evitar a mistura de fezes e urina.

Após o término do experimento, todas as amostras (sobras e fezes) foram secas em estufa de ventilação forçada à temperatura de $65^{\circ} \mathrm{C}$, durante 72 horas. Posteriormente, foram moídas em moinho tipo Willey, com peneira de $1 \mathrm{~mm}$ e estocadas em sacos plásticos para análises laboratoriais, segundo a AOAC (Association of Official Analytical Chemists) para matéria seca (método 930.15; AOAC, 2012) e, com base na MS, proteína bruta (método 968.06, AOAC, 2012), extrato etéreo (954.05; AOAC, 2012), matéria mineral (método 942.05; AOAC, 2012) e teor de fibra em detergente neutro pelo método de VAN SOEST et al. (1991).

Os valores de carboidratos não fibrosos (CNF) foram calculados de acordo com VAN SOEST et al. (1991) e os nutrientes digestíveis totais (NDT), conforme WEISS et al. (1992), a partir das fórmulas: $\mathrm{CNF}=100-(\mathrm{FDN}+\mathrm{PB}+\mathrm{EE}+\mathrm{MM})$ e $\mathrm{NDT}=\mathrm{PBD}+$ $(\mathrm{EEDx} 2,25)+\mathrm{FDND}+\mathrm{CNFD}$, respectivamente.

Utilizou-se o delineamento em blocos completos casualizados nos ensaios de desempenho e comportamento, com quatro tratamentos e cinco blocos, definidos de acordo com o peso inicial dos animais, segundo o modelo: $Y i j k=M+B_{i}+D j+E_{i j}$, em que: $\mathrm{M}=$ média; $\mathrm{B}$ : efeito do bloco $(\mathrm{i}=1$ a 5$) ; \mathrm{D}_{\mathrm{i}}$ $=$ efeito da dieta $(\mathrm{j}=1 \mathrm{a} 4) ; \mathrm{E}_{\mathrm{ij}}=$ efeito aleatório. $\mathrm{O}$ bloco foi incluído como efeito aleatório. $\mathrm{O}$ ensaio de digestibilidade foi realizado em quadrado latino, utilizando-se quatro animais, quatro tratamentos e quatro períodos, por meio do modelo: Yijk $=\mathrm{M}+\mathrm{T}_{\mathrm{i}}+\mathrm{V}_{\mathrm{j}}+\mathrm{P}_{\mathrm{k}}+\mathrm{E}_{\mathrm{ijk}}$, em que: $\mathrm{M}=$ média; $\mathrm{T}_{\mathrm{i}}=$ efeito do tratamento $(\mathrm{i}=1$ a 4$) ; \mathrm{V}_{\mathrm{j}}=$ efeito do animal $(\mathrm{j}=1$ a 4$) ; \mathrm{P}_{\mathrm{k}}=$ efeito do período $(\mathrm{k}=1$ a 4$) ; \mathrm{E}_{\mathrm{ijk}}=$ efeito aleatório.

As variáveis foram analisadas usando o procedimento "MIXED” do SAS (1999). As médias foram obtidas pelo comando LSMEANS. Os efeitos dos teores de inclusão de glicerina bruta nas dietas foram avaliados por meio de polinômios ortogonais linear e quadrático. Os efeitos foram declarados significativos quando $\mathrm{P}<0,05$.

\section{RESULTADOS E DISCUSSÃO}

Os consumos de matéria seca (CMS), proteína bruta $(\mathrm{CPB})$, fibra em detergente neutro 
(CFDN) e extrato etéreo (CEE) dos animais em confinamento não foram afetados $(\mathrm{P}>0,05)$ pela inclusão de glicerina bruta (Tabela 2). Os consumos médios de matéria seca $\left(0,591 \pm 0,09 \mathrm{~kg}\right.$ de $\left.\mathrm{MS} \mathrm{dia}^{-1}\right) \mathrm{e}$ proteína $\left(0,081 \pm 0,015 \mathrm{~kg} \mathrm{dia}^{-1}\right)$ dos cabritos durante o período de confinamento neste estudo estão de acordo com o NRC (2007), cujas recomendações são: $0,570 \mathrm{~kg}$ de MS $\operatorname{dia}^{-1}$ e $0,091 \mathrm{~kg}$ de PB dia ${ }^{-1}$, respectivamente, para animais Boer de $15 \mathrm{~kg}$ de peso vivo com ganhos de peso de 100 gramas dia $^{-1}$.

A ausência de efeito dos diferentes teores de glicerina bruta sobre o CMS pode estar relacionado à semelhança na composição química das dietas experimentais. Adicionalmente, as dietas experimentais, mesmo com a substituição parcial do milho, apresentaram teores de NDT também muito próximos, o que contribuiu para os resultados encontrados.

Normalmente, a glicerina bruta é utilizada na dieta substituindo fontes energéticas, no caso do presente estudo, o milho. Sendo a glicerina coproduto da produção do biodiesel, sua composição pode ser variada, devendo este fator ser considerado no momento de formulação da dieta. Esses fatores, estando dentro do padrão considerado seguro, tornam a glicerina viável para uso na dieta de ruminantes, sem afetar o consumo, como observado no estudo.

O CFDN não sofreu efeitos $(\mathrm{P}=0,08)$ da inclusão da glicerina bruta. Esse resultado era esperado e é reforçada por CARVALHO et al. (2011) ao afirmarem que a FDN, muito embora não o único, é um dos principais fatores que interferem sobre o consumo de determinada ração. Como observado na tabela 1, o teor de FDN das dietas experimentais foram similares, resultando, portanto, em consumos equivalentes. Da mesma forma, o CEE não foi influenciado $(\mathrm{P}>0,05)$ pela adição da glicerina bruta na dieta. Isto se deve ao CMS equivalente entre os tratamentos e a semelhança do teor de EE entre as dietas.

$\mathrm{O}$ ganho de peso médio diário (GMD) e o peso final (PF) dos animais ao término do confinamento também não sofreram efeitos $(\mathrm{P}>0,05)$ da inclusão da glicerina bruta. Isto se deve à grande semelhança entre as concentrações de energia do milho e do glicerol e ao fato de o consumo de MS e nutrientes não ter sido alterado. A concentração energética do glicerol é da ordem de 1,98 a 2,29Mcal $\mathrm{kg}^{-1}$, próxima à concentração encontrada no amido do milho (SCHRÖDER \& SÜDEKUM, 1999). Este fato

Tabela 2 - Consumo, digestibilidade e desempenho de cabritos alimentados com dietas contendo glicerina bruta.

\begin{tabular}{|c|c|c|c|c|c|c|c|}
\hline \multirow{2}{*}{ Variáveis ${ }^{1}$} & \multicolumn{6}{|c|}{ 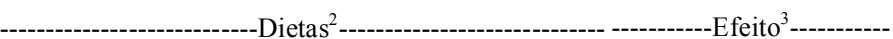 } & \multirow{2}{*}{$\mathrm{CV}^{4}(\%)$} \\
\hline & G0 & G4 & G8 & G12 & $\mathrm{L}$ & Q & \\
\hline ------"------"- & & $\ldots$ & sumo & & & & 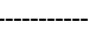 \\
\hline \multicolumn{8}{|l|}{ MS } \\
\hline $\mathrm{g} \mathrm{dia}^{-1}$ & 583,7 & 650,6 & 537,1 & 587,6 & 0,51 & 0,93 & 17,00 \\
\hline$\%$ do PV & 2,95 & 3,26 & 2,75 & 3,03 & 0,69 & 0,93 & 12,67 \\
\hline g UTM $^{-0,75}$ & 62,25 & 68,76 & 59,09 & 63,45 & 0,60 & 0,98 & 12,83 \\
\hline $\mathrm{PB}, \mathrm{g} \mathrm{dia}^{-1}$ & 75,00 & 94,38 & 72,80 & 81,72 & 0,87 & 0,54 & 19,10 \\
\hline FDN, $\mathrm{g} \mathrm{dia}^{-1}$ & 205,8 & 223,1 & 186,0 & 190,1 & 0,13 & 0,90 & 16,26 \\
\hline $\mathrm{EE}, \mathrm{g} \mathrm{dia}^{-1}$ & 15,78 & 18,88 & 14,22 & 15,30 & 0,19 & 0,22 & 17,81 \\
\hline & & --------D & bilidad & & & & \\
\hline $\mathrm{MS}, \%$ & 76,44 & 77,95 & 80,83 & 79,99 & 0,17 & 0,54 & 5,21 \\
\hline $\mathrm{PB}, \%$ & 67,49 & 74,60 & 78,32 & 76,08 & 0,21 & 0,33 & 11,04 \\
\hline FDN, \% & 79,18 & 81,83 & 78,60 & 75,56 & 0,25 & 0,30 & 7,20 \\
\hline $\mathrm{EE}, \%$ & 87,26 & 84,24 & 83,90 & 88,82 & 0,33 & $<0,01$ & 3,73 \\
\hline Peso inicial, $\mathrm{kg}$ & 17,11 & 17,38 & 16,80 & 17,03 & 0,65 & 0,96 & 12,66 \\
\hline Peso final, $\mathrm{kg}$ & 22,50 & 22,80 & 21,54 & 21,80 & 0,36 & 0,98 & 13,30 \\
\hline $\mathrm{GMD}, \mathrm{g} \mathrm{dia}^{-1}$ & 105,0 & 106,0 & 90,00 & 93,00 & 0,28 & 0,90 & 27,39 \\
\hline $\mathrm{CA}, \mathrm{kg} \mathrm{kg}^{-1}$ de ganho & 5,69 & 6,38 & 6,23 & 6,62 & 0,24 & 0,76 & 17,50 \\
\hline
\end{tabular}

${ }^{1} \mathrm{MS}$ : matéria seca, PB: proteína bruta, FDN: fibra em detergente neutro, EE: extrato etéreo, GMD: ganho médio diário, CA: conversão alimentar.

${ }^{2} \mathrm{G} 0, \mathrm{G} 4$, G8, G12: adição de $0,4,8$ e $12 \%$ de glicerina bruta na dieta, respectivamente. ${ }^{3}$ Valor de P para o teste de polinômio ortogonal, linear (L) ou quadrático (Q).

${ }^{4}$ Coeficiente de variação. 
pode ser confirmado com o teor de NDT das dietas (Tabela 1) que apresentou valores de 87,45; 89,23; 88,05 e $87,15 \%$ para os tratamentos G0, G4, G8 e G12, respectivamente.

O ganho de peso encontrado neste trabalho (valor médio de $98,5 \mathrm{~g} \mathrm{dia}^{-1}$ ) foi inferior ao preconizado pelo NRC (2007) para caprinos Boer. Esses valores são justificados pela presença de um surto de verminose que acometeram os animais no início do período experimental e também pelo fato de os animais serem mestiços, o que certamente alterou o GMD predito NRC (2007).

A conversão alimentar (CA) não apresentou variação $(\mathrm{P}>0,05)$ com a inclusão da glicerina bruta. A igualdade encontrada para a conversão alimentar entre os tratamentos se deve ao fato de o CMS e GMD também não terem apresentado diferença significativa.

A digestibilidade da MS, PB e FDN não foram afetadas $(\mathrm{P}>0,05)$ pela inclusão da glicerina bruta (Tabela 2). Consumo similar para as dietas avaliadas, bem como a similaridade nos ingredientes utilizados poderia explicar a ausência de diferença na digestibilidade dos nutrientes entre dietas. Entretanto, a digestibilidade do EE apresentou comportamento quadrático $(\mathrm{P}<0,01)$. Na composição química das dietas avaliadas, há ligeiro decréscimo do teor do EE na MS das rações com a inclusão da glicerina bruta, o que pode ter ocasionado tal efeito. Adicionalmente, essa variação na digestibilidade do EE pode estar associada à composição da glicerina bruta, que substituiu parcialmente o milho das dietas, ou da fonte de matéria-prima ou do processo de produção do biodiesel, que deu origem à glicerina utilizada, a qual também pode ter interferido na digestibilidade do EE (FARIAS et al., 2012)

Não houve efeito dos tratamentos para a DPB, devido à ausência de alteração no CMS e também pelo fato de as dietas serem isonitrogenadas. Este fato pode ter contribuído para a ausência de efeito na digestibilidade da fibra. GELINSKI et al. (2000) correlacionam uma boa DFDN com a presença de proteína ou nitrogênio não proteico no ambiente ruminal, enquanto que MALLMANN et al. (2006) atribui a baixa digestibilidade da FDN de fenos de baixa qualidade a valores pobres de $\mathrm{PB}$, os quais limitam a proliferação das bactérias celulolíticas, devido à baixa disponibilidade de ácidos graxos de cadeia ramificada. Estes ácidos graxos, por sua vez, são resíduos do metabolismo de organismos proteolíticos e, ao mesmo tempo, substratos para a proliferação de organismos celulolíticos.

Os dados deste estudo referentes à digestibilidade de MS, PB e FDN estão de acordo com os estudos realizados por ÁVILA-STAGNO et al. (2012), que não encontraram diferença destas variáveis ao avaliarem a adição de até $21 \%$ de glicerina bruta na dieta de cordeiros confinados. Segundo os autores, a adição de glicerina bruta pode aumentar a digestibilidade da matéria seca e nutrientes dos animais alimentados basicamente com forragem, entretanto, tem impacto mínimo ou nulo na digestibilidade de animais alimentados com dietas com alta proporção de concentrados. Essas variações podem ainda estar relacionadas à grande variação na composição da glicerina produzida, principalmente no que diz respeito aos compostos contaminantes como sais, que podem reduzir o consumo e a digestibilidade dos nutrientes.

O tempo de ingestão em minutos dia $^{-1}$, minutos $\mathrm{g}^{-1}$ de MS e minutos $\mathrm{g}^{-1}$ de FDN aumentou linearmente $(\mathrm{P}<0,01)$ conforme o nível de inclusão da glicerina bruta na MS da dieta (Tabela 3). É provável que esses resultados possam ser explicados pelo poder de seletividade dos componentes físicos da dieta inerente à espécie caprina, uma vez que o aumento do tempo dedicado à ingestão não se traduziu em aumento no consumo de MS. É provável que, devido às propriedades higroscópicas da glicerina (ELAM et al., 2008), as dietas contendo maior percentagem deste ingrediente exijam maior tempo de salivação do bolo alimentar antes da deglutição.

Os tempos de ruminação em minutos/dia, minutos/grama de MS e minutos/grama de FDN não foram afetados $(\mathrm{P}>0,05)$ pelos tratamentos (Tabela 3). De acordo com CARVALHO et al. (2011), a composição química do alimento, principalmente em termos de FDN, pode influenciar diretamente no comportamento ingestivo de ruminantes, uma vez que este nutriente é o principal fator que interfere no bom funcionamento ruminal. Neste caso, o teor de FDN foi semelhante em todas as dietas, o que favoreceu a ausência de efeito nos resultados relacionados ao tempo de ruminação.

Diante dos resultados, fazem-se necessárias avaliações de novos estudos, principalmente nos aspectos nutricionais dos animais e qualitativos do produto final.

\section{CONCLUSÃO}

Recomenda-se a inclusão em até $12 \%$ de glicerina bruta para cabritos em terminação, desde que seja viável em situações de oscilação do preço do milho, sem alteração nos parâmetros produtivos avaliados.

Ciência Rural, v.45, n.4, abr, 2015. 
Tabela 3 - Comportamento ingestivo de cabritos alimentados com dietas contendo glicerina bruta.

\begin{tabular}{|c|c|c|c|c|c|c|c|}
\hline & \multicolumn{6}{|c|}{ 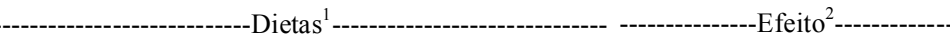 } & \multirow{2}{*}{$\mathrm{CV}(\%)^{3}$} \\
\hline & G0 & G4 & G8 & G12 & $\mathrm{L}$ & Q & \\
\hline \multicolumn{8}{|c|}{ 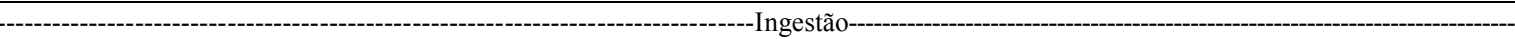 } \\
\hline $\min \mathrm{dia}^{-1}$ & 195,0 & 284,0 & 306,5 & 331,0 & $<0,01$ & 0,52 & 31,1 \\
\hline $\min \mathrm{g}^{-1}$ de MS & 0,32 & 0,44 & 0,62 & 0,56 & $<0,01$ & 0,20 & 41,3 \\
\hline $\min \mathrm{g}^{-1}$ de FDN & 0,79 & 1,09 & 1,53 & 1,56 & 0,01 & 0,43 & 42,2 \\
\hline $\min \operatorname{dia}^{-1}$ & 387,0 & 360,0 & $\begin{array}{c}\text { Rumina } \\
368,5\end{array}$ & 347,0 & 0,40 & 0,92 & 20,8 \\
\hline $\min \mathrm{g}^{-1}$ de $\mathrm{MS}$ & 0,66 & 0,55 & 0,73 & 0,60 & 0,96 & 0,91 & 24,7 \\
\hline $\min g^{-1}$ de FDN & 1,59 & 1,38 & 1,80 & 1,60 & 0,45 & 0,96 & 24,0 \\
\hline \multicolumn{8}{|l|}{ Ocio } \\
\hline Min $\operatorname{dia}^{-1}$ & 850,5 & 787,0 & 773,5 & 733,5 & 0,07 & 0,78 & 14,1 \\
\hline
\end{tabular}

${ }^{1} \mathrm{G} 0, \mathrm{G} 4, \mathrm{G} 8, \mathrm{G} 12$ : adição de $0,4,8$ e $12 \%$ de glicerina bruta na dieta, respectivamente.

${ }^{2}$ Valor de $\mathrm{P}$ para o teste de polinômio ortogonal, linear (L) ou quadrático (Q).

${ }^{3}$ Coeficiente de variação.

\section{AGRADECIMENTOS}

Os autores agradecem à Fundação de Amparo à Pesquisa e Desenvolvimento Científico do Maranhão (FAPEMA) pelo auxílio financeiro (Processo n 00342/12).

\section{REFERÊNCIAS}

AOAC International. Official methods of analysis of AOAC international. Gaithersburg: Association of Analytical Communities, 2012. 3000p.

ÁVILA-STAGNO, J. et al. Effects of increasing concentrations of glycerol in concentrate diets on nutrient digestibility, methane emissions, growth, fatty acid profiles, and carcass traits of lambs. Journal of Animal Science, v.91, p.829-837, 2012. Disponível em: $\quad<$ http://www.journalofanimalscience.org/content/91/2/829. full.pdf + html $>$. Acesso em: maio 2013. doi: 10.2527/jas.20125215 .

AZEVEDO, R.A. et al. Comportamento ingestivo de cordeiros alimentados com torta de macaúba. Arquivo Brasileiro de Medicina Veterinária e Zootecnia, v.65, n.2, p.490-496, 2013. Disponível em: $<$ http://dx.doi.org/10.1590/S0102-09352013000200027>. Acesso em: mar. 2014. doi:10.1590/S0102-09352013000200027.

CARVALHO, G.G.P.C. et al. Comportamento ingestivo em caprinos alimentados com dietas contendo cana-de-açúcar tratada com óxido de cálcio. Revista Brasileira de Zootecnia, v.40, n.4, p.1767-1773, 2011. Disponível em: <http://dx.doi.org/10.1590/ S1516-35982011000800021>. Acesso em: abr. 2014. doi: $10.1590 / \mathrm{S} 1516-35982011000800021$.

DASARI, M.A.P. et al. Low pressure hydrogenolysis of glycerol to propylene glycol. Applied Catalysis General, v.281, p.225-231, 2005. Disponível em: <http://dx.doi.org/10.1016/j. apcata.2004.11.033>. Acesso em: dez. 2013. doi: 10.1016/j. apcata.2004.11.033.

ELAM, N.A. et al. Glycerol from biodiesel production: considerations for feedlot diets. In: PROCEEDINGS OF THE
SOUTHWEST NUTRITION CONFERENCE, 21., 2008, Tempe AZ. Proceedings...Tempe: University of Arizona 2008. p.131.

FARIAS, M.S. et al. Níveis de glicerina para novilhas suplementadas em pastagens: desempenho, ingestão, eficiência alimentar e digestibilidade. Semina: Ciências Agrárias, v.33, p.1177-1188, 2012. Disponível em: <http://www.uel.br/revistas/ uel/index.php/semagrarias/article/viewFile/8017/10854>. Acesso em: maio 2014. doi: 10.5433/1679-0359.2012v33n3p1177.

FDA (Food and Drug Administration). 2006. Code of Federal Regulations. Title 21-Food and Drugs. Vol. 6, 21CFR582.1320. Capturado em 12 jan. 2014. Online. Disponível em: <http:// edocket.access.gpo.gov/cfr_2002/aprqtr/21cfr582.1320.htm>.

GELINSKI, L.A.M. et al. Monensina e uréia de liberação lenta no desempenho de bovinos confinados. Archives of Veterinary Science. v.5, p.137-140, 2000. Disponível em: <http://ojs.c3sl. ufpr.br/ojs/index.php/veterinary/article/viewFile/3898/3138>. Acesso em: nov. 2013.

KREHBIEL, C.R. Ruminal and physiological metabolism of glycerin. Journal of Animal Science, v.86, p.392, 2008. Disponível em: $\quad<$ http://adsa.asas.org/meetings/2008/abstracts/0392.PDF>. Acesso em: 19 jan. 2013.

LAGE, J.F. et al. Carcass characteristics of feedlot lambs fed crude glycerin contaminated with high concentrations of crude fat. Meat Science, v.96, p.108-113, 2014. Disponível em: $<$ http://dx.doi. org/10.1016/j.meatsci.2013.06.020>. Acesso em: dez. 2014. doi: 10.1016/j.meatsci.2013.06.020.

MALLMANN, G.M. et al. Consumo e digestibilidade de feno de baixa qualidade suplementado com nitrogênio não proteico em bovinos. Pesquisa Agropecuária Brasileira, v.41, n.2, p.331337, 2006. Disponível em: <http://dx.doi.org/10.1590/S0100204X2006000200019>. Acesso em: out. 2013. doi: 10.1590/ S0100-204X2006000200019.

NATIONAL RESEARCH COUNCIL (NRC). Nutrient requirements of small ruminants: sheep, goats, cervids, and new world camelids. Washington, D.C.: National Academic, 2007. 292p. 
STATISTICAL ANALYSES SYSTEM (SAS). SAS/STATTM. SAS user's guide for windows environment. version 6. 11.ed. Cary, 1999. $1686 \mathrm{p}$.

SCHRÖDER, A.; SÜDEKUM, K.H. Glycerol as a by-product of biodiesel production in diets for ruminants. In: INTERNATIONAL RAPESEED CONGRESS, 10, 1999, Gosford. Proceedings... Gosford: New Horizons for an Old Crop The Regional Institute, 1999. p. 241

VAN SOEST, P.J. et al. Methods for dietary fiber, neutral detergent fiber, and nonstarch polysaccharides in relation to animal nutrition. Journal of Dairy Science, v.74, p.3583-3597, 1991. Disponível em: <http://download.journals.elsevierhealth.com/ pdfs/journals/00220302/PIIS0022030291785512.pdf>. Acesso em: abr. 2014.

WEISS, W.P. et al. A therotically-based model for predicting total digestible nutrient values of forage and concentrates. Animal Feed Science and Technology, v.39, n.1/2, p.95110, 1992. Disponível em: <http://dx.doi.org/10.1016/03778401(92)90034-4>. Acesso em: maio 2014. doi: 10.1016/03778401(92)90034-4. 